\title{
Status of the present ATLAS RPC system and overview towards HL-LHC
}

\author{
Gian Luigi Alberghi ${ }^{a *}$, on behalf of the ATLAS Muon Collaboration \\ ${ }^{a}$ INFN Bologna, via Irnerio 46, 40126, Bologna, Italy \\ e-mail: alberghi@bo.infn.it
}

July 2, 2018

\begin{abstract}
The Resistive Plate Chamber (RPC) system covers the barrel region of the ATLAS muon spectrometer [1] in the pseudo-rapidity range $|\eta|<1.05$, with six independent detector layers exclusively providing the first level trigger signal and the track coordinate in the non-bending plane of the muon candidates. The system is designed to operate up to the nominal Large Hadron Collider luminosity $\left(\mathcal{L}=10^{34} \mathrm{~cm}^{-2} \mathrm{~s}^{-1}\right)$ which has been already exceeded thanks to the excellent performance of the collider. The experience in operating the present RPC system, up to the maximum instantaneous luminosity of $2.06 \times 10^{34} \mathrm{~cm}^{-2} \mathrm{~s}^{-1}$ reached in 2017 , is reported. The performance of the system, in the severe background and pileup conditions of the last data taking period, is presented together with the improved tools implemented in order to have an effective monitoring of the detector status. The plans to successfully operate the present system during the HL-LHC phase are also introduced.
\end{abstract}

Keywords: Resistive-plate chambers, Muon spectrometers

\footnotetext{
${ }^{*}$ Corresponding author

Copyright 2018 CERN for the benefit of the ATLAS Collaboration.

CC-BY-4.0 license.
} 


\section{Contents}

1 Introduction $\quad 2$

2 The ATLAS Resistive Plate Chambers 2

3 Detector Status and Operation 3

4 Overview towards HL-LHC $\quad 8$

5 Conclusions $\quad 8$

\section{Introduction}

In 2017 the Large Hadron Collider has provided proton-proton collisions at a center of mass energy of $13 \mathrm{TeV}$ with instantaneous luminosities up to $2.06 \times 10^{34} \mathrm{~cm}^{-2} \mathrm{~s}^{-1}$, totaling an integrated luminosity of $50.4 \mathrm{fb}^{-1}$. This document presents the performance and the operational experience of the detector in this period of time and a brief overview of the upgrade activities for the High Luminosity phase of LHC. The document is organized as follows: after a short description of the ATLAS Resistive Plate Chambers (RPCs) in Section 2, the detector status and performance with details of operational experience are presented (Section 3). The improvements and upgrade activities in view of the High-Luminosity LHC phase are described in Section 4, finally some concluding remarks and outlook are given in Section 5 .

\section{The ATLAS Resistive Plate Chambers}

RPCs [2] provide the barrel region of the ATLAS detector [3] with an independent muon trigger and a two-coordinate measurement. The chambers, arranged in three concentric double layers (middle confirm, pivot, outer confirm) are operated in a strong magnetic toroidal field and cover a surface area of about $4000 \mathrm{~m}^{2}$. A low $p_{T}(<10 \mathrm{GeV})$ trigger requires a projective coincidence between hits in the pivot and the middle confirm layer while high $p_{T}$ triggers require an additional hit in the outer confirm layer. An ATLAS RPC chamber is made of two layers, each with two $2 \mathrm{~mm}$ thick bakelite laminate plates and providing two $\eta$ and two $\phi$ readout strip planes (see Fig.1). Made of phenolic and melaminic resins, the plates are kept apart at $2 \mathrm{~mm}$ by insulating spacers, enclosing a gas volume filled with a mixture of $C_{2} H_{2} F_{4}(94.7 \%)-C_{4} H_{10}(5 \%)-S F_{6}(0.3 \%)$. The external surface of the plates is coated with a thin layer of graphite paint to allow a uniform distribution of the high voltage along the plates. The smoothness of the inner 


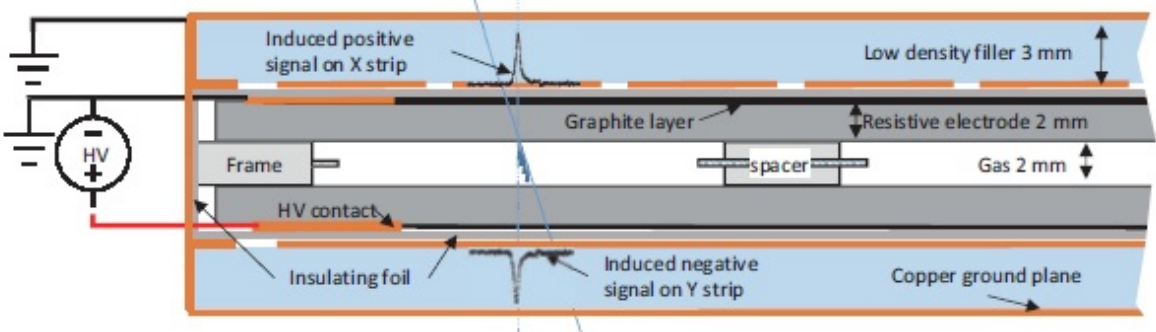

Figure 1: A single gap RPC structure

surfaces is enhanced by means of a thin layer of linseed oil. The high voltage (HV) working point is chosen to be at $9.6 \mathrm{kV}$ at a temperature of $24^{\circ} \mathrm{C}$ and a pressure of 970 mbar. In these conditions the RPCs work in saturated avalanche mode, inducing, for a minimum ionizing particle, a prompt charge of about $1 \mathrm{pC}$ on the pick-up strips and delivering an average total charge of $30 \mathrm{pC}$ in the gas. The discharge electrons drift in the gas and the signal, induced on pick-up copper strips, is read out via capacitive coupling, and detected by the front-end electronics. Read-out strips have a typical width of $\sim 30 \mathrm{~mm}$ and are grouped in two $(\eta$ and $\phi)$ read-out panels with strips orthogonal to each other. Custom front-end electronics amplifies, discriminates and converts the detector signals to ECL standard. These signals are transmitted to the on-detector trigger electronics [4] which, by requiring appropriate coincidences in the $\eta$ and $\phi$ detector layers, provide ATLAS with a Level 1 trigger decision along with the detector data for accepted events.

\section{Detector Status and Operation}

\section{RPC performance}

In 2017 LHC provided proton-proton collisions at center of mass energy $\sqrt{s}$ $=13 \mathrm{TeV}$ with $25 \mathrm{~ns}$ bunch spacing. The peak instantaneous luminosity was $2.06 \times 10^{34} \mathrm{~cm}^{-2} \mathrm{~s}^{-1}$ and the peak number of collisions per bunch crossing (pile-up) was about 80. The 2017 full dataset is made of a delivered integrated luminosity of $50.4 \mathrm{fb}^{-1}$ a recorded integrated luminosity of $46.8 \mathrm{fb}^{-1}$ out of which $43.8 \mathrm{fb}^{-1}$ were considered good for physics analysis. The 2017 data taking has been very successful for the RPC system which has been running with a fraction of up-time and good data quality efficiency of $97.8 \%$. The number of gas volumes either disconnected or operated not at nominal conditions has been around 6\% with 239 total disconnected gaps (out of 3714), out of which 152 were disconnected on chamber, 83 on rack (partially recoverable during Technical Stops) and 4 kept at low voltage for recovery 
due to bad gas supply or too high currents. During 2017 three main hardware issues were responsible for the RPC down time: a power crate failure affecting one (out of 100) Caen Easy crate; a Caen Power Supply mainframe failure, stopping one RPC quadrant; some power supply glitches, due to the muon common system infrastructure, causing the main part of the RPC down time.

\section{Detector Control System}

ATLAS RPCs are monitored and operated through the Detector Control System (DCS) which is coherently integrated in the muon system so that a single shifter can operate and monitor all the muon sub-detectors. Several automatic monitoring and control tools to simplify the detector operation and optimize the data taking conditions. One important feature is the full automatic control of the HV settings, which are automatically adjusted in order to follow the different LHC beam phases from injection, stable beams, to the final dump; to compensate for the local changes of the environmental conditions (mainly local temperature and atmospheric pressure); to automatically check the individual gas-gaps currents, recalibrate at each end of fill the pedestals ad finally to provide an online measurement of the cavern background rates and an instantaneous luminosity The DCS is in charge of safely operating and monitoring the detector power system including the detector HV and LV supply. In the RPC DCS [5], a large number of settings (DAC $\sim 4000)$ and monitoring (ADC 6500) channels have been integrated into the system in order to optimize the detector performance and allow a fine monitoring at the level of the single individual RPC gas gap $(\sim 3600)$. The remaining ADC channels are used to monitor with high granularity the current draw of the front end electronics and RPC gas and environmental sensors (temperature, atmospheric pressure, relative humidity and gas flow). The ability to control by tuning thresholds, and monitoring the current of each RPC gap has shown to be very powerful for the detector operation both for tracing problems and fine tune the detector. This is particularly important as the RPC performance and aging is strongly related to the environmental parameters, namely the temperature $(T)$, the atmospheric pressure $(P)$, and the relative humidity. The gas gain, the noise rate and the dark current of the chamber depend on these parameters following the formula: $V_{\text {appl }}=V_{\text {eff }} \cdot\left(T_{0} / T\right) \cdot\left(P / P_{0}\right)$ where $V_{\text {appl }}$ is the applied voltage, $T, P$ are the environment measurements and $T_{0}, P_{0}, V_{\text {eff }}$ are the reference environmental values and HV settings.

\section{Technical Issues}

During the years of operation, some gas volume inlets have developed fractures or complete breakups. This issue is currently affecting about $6 \%$ of 


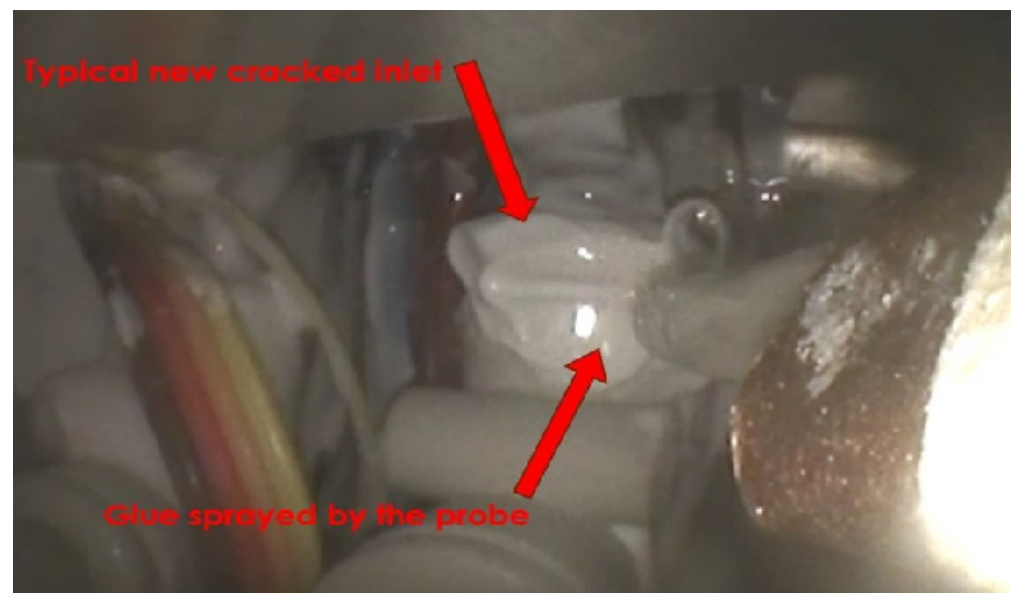

Figure 2: A partial crack of the gas inlet sealed by sprayed glue

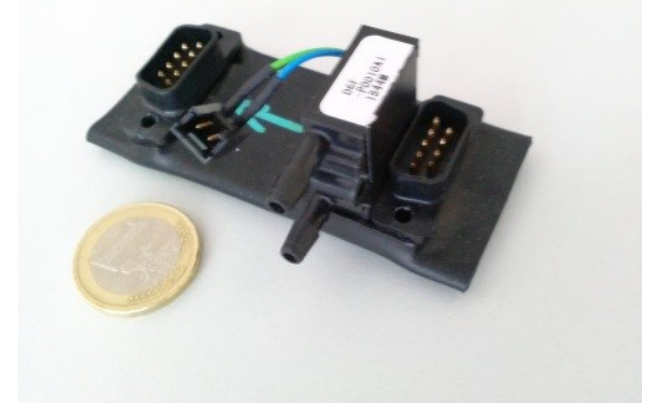

Figure 3: A flowmeter used for single chamber gas output online monitoring

gas gaps and is intensely investigated, as it is responsible for most of the RPCs inefficiency. As most of the volumes can be operated normally, a repair campaign is ongoing. The type of intervention depends on the crack features: if the inlet is completely broken a substitution procedure must be done. On the other hand, if the inlet has developed only a partial fracture, it is possible to intervene by spraying glue on the break, building a sealing membrane (see Fig.2). In the past years many tests both on the procedure for the glue spraying and on the choice of the particular glue to be used have undergone, so that now the procedure is very solid and the results very reliable. In order to provide online monitoring of the gas flow through individual chambers, starting on the end of 2016, 1200 flowmeters (see Fig.3) have been installed at the gas output of the RPCs. The flowmeter readings are fully integrated in the DCS through ADCs (see Fig.4) and are monitored with automatic alarms. It is thus possible to promptly identify the single chamber leaks at a very early stage, allowing a rapid intervention at a time 


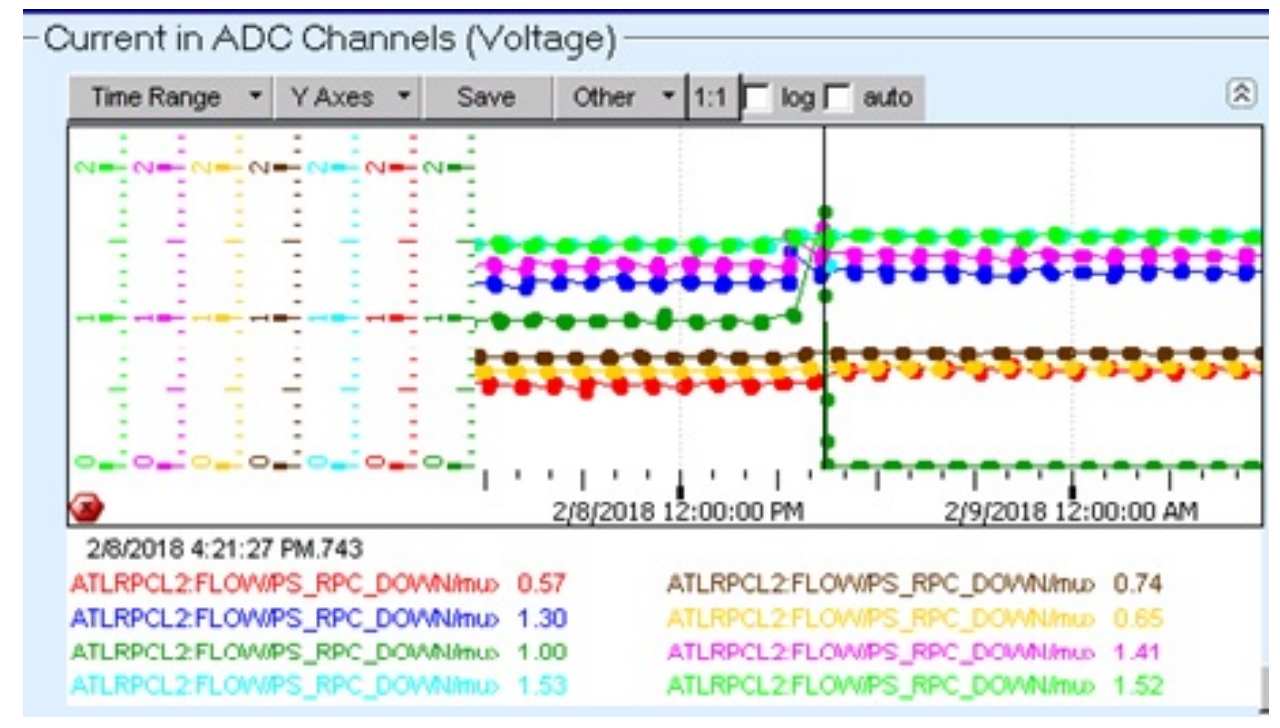

Figure 4: ADC readings of chamber's output flow through gas flowmeters, each color representing the flow of a single RPC

when the reparation is easier. A good news for the year 2018 comes from the fact that, taking into account the experience from previous years when a large increase of the gas leaks after the system restart (after the winter shutdown) was observed, the decision not to stop the gas system was taken. At the 2018 operation restart no increase in the gas leak was observed (see Fig.5), allowing for the possibility to start the repair campaign from a better portion than last years.

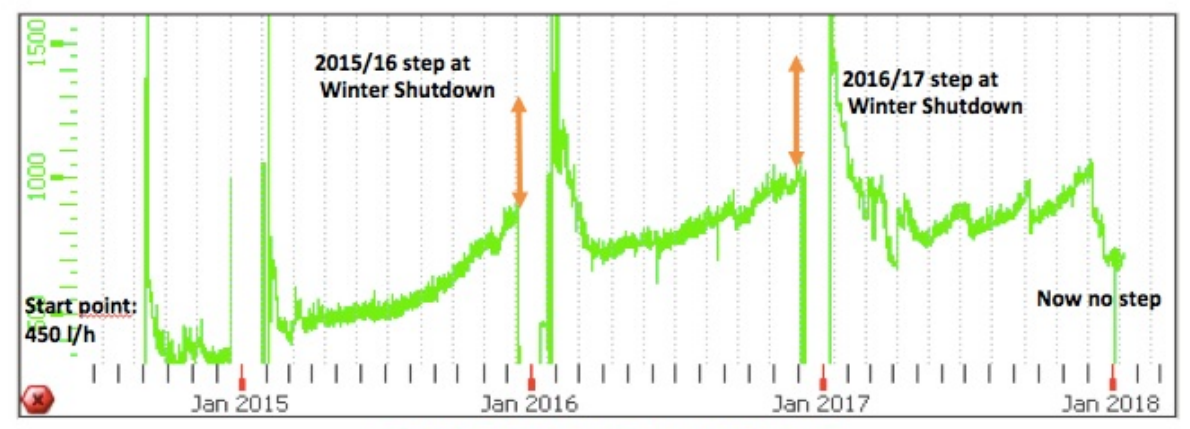

Fresh Gas Consumption loss rate

Figure 5: Fresh gas consumption rate since July 2014 


\begin{tabular}{|c|c|c|c|c|c|c|c|c|c|c|c|c|c|c|c|c|c|c|c|c|c|}
\hline \multirow{2}{*}{$\begin{array}{l}\text { Sector } \\
\boldsymbol{\Psi}_{\mathrm{kd} \text {. }}\end{array}$} & \multicolumn{20}{|c|}{$\mathrm{RPC}$ unit Id. along Z direction } & \multirow[b]{2}{*}{ Average } \\
\hline & -6.2 & -6.1 & -5.0 & -4.0 & -3.2 & -3.1 & -2.2 & -2.1 & -1.2 & -1.1 & 1.1 & 1.2 & 2.1 & 2.2 & 3.1 & 3.2 & 4.0 & 5.0 & 6.1 & 6.2 & \\
\hline 01.01 & 342 & 280 & 301 & 225 & 145 & 114 & 128 & 101 & & 61 & 61 & 71 & 113 & 122 & 127 & 136 & 214 & 276 & 269 & 285 & 180 \\
\hline 01.02 & 293 & 281 & 303 & 218 & 159 & 133 & 129 & 143 & & 76 & 71 & 75 & 127 & 143 & 140 & 148 & 215 & 295 & 278 & 297 & 188 \\
\hline 2 & 168 & 204 & 188 & 138 & 109 & 90 & $\pi$ & 63 & 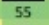 & 56 & 45 & 48 & 61 & 79 & 94 & 104 & 140 & 207 & 196 & 152 & 115 \\
\hline 03.01 & 297 & 296 & 281 & 198 & 148 & 128 & 119 & 119 & 68 & ต7 & 65 & 71 & 131 & 125 & 114 & 125 & 207 & 329 & 268 & 290 & 177 \\
\hline 03.02 & 300 & 243 & $2 \pi$ & 210 & 151 & 129 & 155 & 122 & 85 & 75 & 75 & 70 & 122 & 127 & 152 & 132 & 207 & 315 & 243 & 299 & 179 \\
\hline 4 & 112 & 166 & 158 & 151 & 101 & 83 & 65 & 77 & 41 & 53 & 46 & 41 & 73 & 68 & 92 & 108 & 160 & 196 & 175 & 112 & 101 \\
\hline 05.01 & 171 & 173 & 263 & 138 & 105 & 102 & 140 & 127 & 68 & 60 & 60 & 69 & 124 & 177 & 102 & 137 & 185 & 290 & 173 & 171 & 149 \\
\hline 05.02 & 227 & 198 & 237 & 158 & 109 & 105 & 136 & 143 & 77 & 61 & 63 & 71 & 111 & 136 & 108 & 141 & 200 & 267 & 255 & 282 & 159 \\
\hline 6 & 175 & 186 & 208 & 163 & 105 & 95 & 90 & 95 & 77 & 59 & 52 & 59 & 84 & 81 & 106 & 124 & 189 & 200 & 227 & 167 & 131 \\
\hline 07.01 & 305 & 263 & 288 & 191 & 154 & 129 & 131 & 114 & & 76 & 78 & & 122 & 139 & 124 & 148 & 185 & 261 & 268 & 305 & 183 \\
\hline 07.02 & 327 & 258 & 216 & 203 & 141 & 112 & 129 & 108 & & 74 & $\pi$ & & 105 & 114 & 112 & 152 & 184 & 278 & 276 & $279^{\circ}$ & 175 \\
\hline 8 & 146 & 196 & 195 & 161 & 103 & 85 & 80 & 70 & 50 & 57 & 54 & 54 & 67 & 74 & 85 & 108 & 168 & 196 & 194 & 156 & 118 \\
\hline 09.01 & 319 & 246 & 301 & 206 & 155 & 117 & 149 & 119 & & 46 & 64 & & 106 & 134 & 124 & 135 & 197 & 283 & 262 & 297 & 181 \\
\hline 09.02 & 347 & 258 & 287 & 205 & 143 & 95 & 107 & 103 & & 58 & 67 & & 99 & 112 & 95 & 137 & 188 & 285 & 265 & 292 & 174 \\
\hline 10 & 174 & 201 & 207 & 147 & 99 & 86 & 68 & 71 & 46 & 43 & 41 & 50 & 64 & 69 & 80 & 103 & 148 & 193 & 201 & 170 & 115 \\
\hline 11.01 & 308 & 244 & 237 & | $157 \mid$ & | $97 \mid$ & 84 & 81 & 87 & & 40 & 43 & & 83 & 94 & 92 & 94 & $\mid 148$ & 227 & 215 & 278 & 132 \\
\hline 11.02 & 196 & 193 & 157 & 105 & 78 & 66 & 55 & 57 & & 33 & 31 & & 50 & 62 & 58 & 71 & 98 & 151 & 160 & 185 & 98 \\
\hline 12 & & & & & & 80 & 81 & 66 & 51 & 36 & 36 & $\overline{51}$ & 75 & 87 & 80 & & & & & & 64 \\
\hline 13.01 & 291 & 278 & 253 & & 140 & 102 & 96 & 84 & 43 & 41 & 47 & 50 & 87 & 95 & 99 & 123 & & 249 & 263 & 319 & 149 \\
\hline 13.02 & 299 & 264 & 262 & & 104 & 97 & 105 & 86 & 49 & 48 & 50 & 56 & 93 & 103 & 97 & 110 & & 252 & 227 & 294 & 146 \\
\hline 14 & & & & & & 142 & 68 & 64 & 52 & 41 & 40 & 49 & 63 & 68 & 136 & & & & & & 76 \\
\hline 15.01 & 196 & 221 & 148 & 113 & 76 & 71 & 67 & 49 & & 38 & 36 & & 50 & 59 & 86 & 87 & 104 & 156 & 173 & 196 & 104 \\
\hline 15.02 & 183 & 159 & 246 & 164 & 116 & 98 & 103 & 75 & & 44 & 43 & & 75 & 106 & 112 & 107 & 158 & 248 & 159 & 183 & 133 \\
\hline 16 & 173 & 214 & 216 & 173 & 108 & 89 & 54 & 75 & $\overrightarrow{56}$ & 59 & 50 & 50 & 77 & 54 & 87 & 103 & 177 & 209 & 208 & 154 & 124 \\
\hline & & & & & & & & & & & & & & & & & & & & & \\
\hline Average & 229 & 223 & 234 & 167 & 118 & 101 & 96 & 88 & 56 & 52 & 51 & 56 & 86 & 97 & 103 & 118 & 171 & 240 & 221 & 221 & 137 \\
\hline
\end{tabular}

Figure 6: Averaged RPC hit rates in BM chambers measured in Run1 and extrapolated (including a safety factor of two) to a luminosity of $7.5 \times 10^{34}$ $\mathrm{cm}^{-2} \mathrm{~s}^{-1}$. The plot shows rates in $\mathrm{Hz} / \mathrm{cm}^{2}$ as a function of the $\phi$ sector (vertical axis) and the station number along $\mathrm{Z}$ (horizontal axis). The rates have been derived form detector currents by applying a conversion factor of $30 \mathrm{pC} /$ hit $[8]$

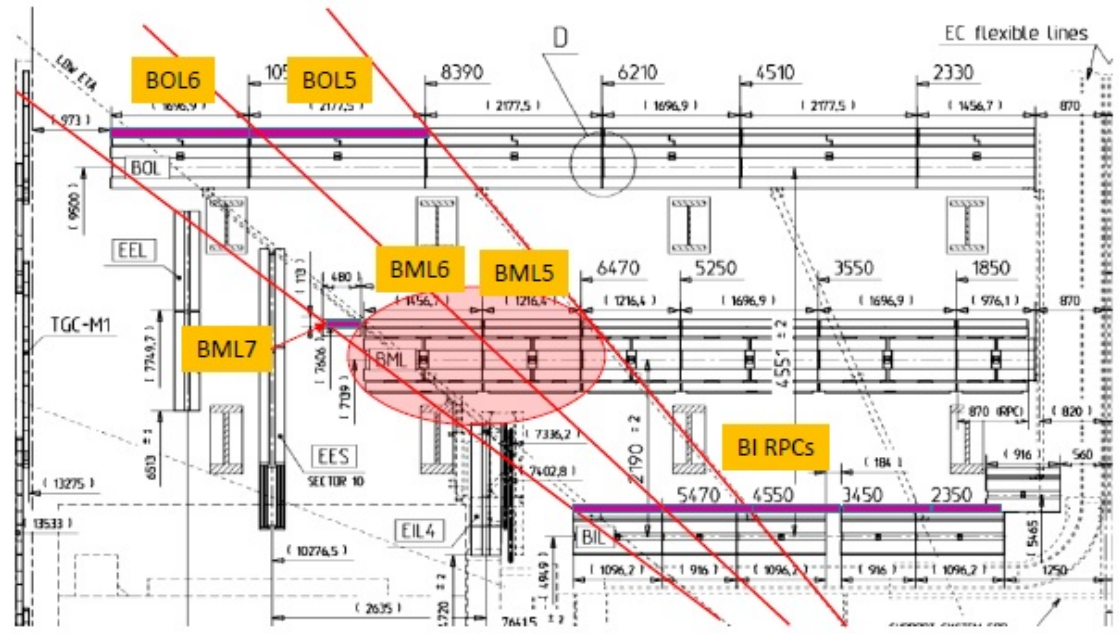

Figure 7: The region affected by a loss of efficiency in the BML6 and BML6 chambers [8] 


\section{Overview towards HL-LHC}

ATLAS RPCs are certified for 10 years of operation at a luminosity of $10^{34} \mathrm{~cm}^{-2} \mathrm{~s}^{-1}$ with a rate of $100 \mathrm{~Hz} \mathrm{~cm}{ }^{-2}$ for a total integrated charge of $0.3 \mathrm{C} \mathrm{cm}^{-2}$. The counting rates at the High Luminosity phase of LHC will exceed this limit, for example the rate at $|\eta|=1$ will be about $300 \mathrm{~Hz}$ $\mathrm{cm}^{-2}$ (see Fig.6). Moreover the extent of the running time from 10 years to 30 years will bring the integrated charge to $0.48 \mathrm{C} \mathrm{cm}^{-2}$ at $|\eta|=1$ for an integrated luminosity of $4000 \mathrm{fb}^{-1}$. As a consequence the charge per count of $30 \mathrm{pC} /$ hit must be reduced in order to compensate the increase in the rate (see [8]). This would nonetheless lower the single-gap efficiency of $15 \%$ on average and of $35 \%$ at large $\eta$. The BIS $7 / 8$ upgrade project and the BI upgrade project are presented in $[6,7]$, so the focus here is on the $\mathrm{BO}$ refurbishment project. The basic idea is that the reduction of trigger efficiency at $|\eta|>0.8$ can be recovered by refurbishing a limited number of BO chambers by replacing the front-end electronics and the readout strip panels, as shown in Fig.7. This can be done during the long shutdown of 2019-2020.

\section{Conclusions}

The ATLAS RPCs have very successfully operated during 2017, maintaining high efficiency and high data quality over the whole period. A few technical issues have been promptly tackled and absorbed by the system redundancy without considerable effects on the data taking as the RPCs are the only system with trigger capability in the barrel region of the muon spectrometer. The consolidation and upgrade works will ensure the ATLAS RPCs will be able to exploit at best the luminosity delivered by the Large Hadron Collider in the next years.

\section{References}

[1] ATLAS Collaboration, The ATLAS Experiment at the CERN Large Hadron Collider JINST 3, S08003 (2008)

[2] R. Santonico R. Cardarelli, Development of resistive plate counters, NIM A, 187 (1981) 377.

[3] ATLAS Muon Collaboration, ATLAS Muon Spectrometer Technical Design Report', CERN/LHCC/97-22 (1997)

[4] F. Anulli et al., The Level-1 Trigger Muon Barrel System of the ATLAS experiment at CERN, JINST 4 (2009) P04010 
[5] A. Polini, Design and Performance of the Detector Control System of the ATLAS Resistive-Plate-Chamber Muon Spectrometer, Nucl. Instrum. Meth. A, 661 (2012) S15

[6] A.Polini, these proceedings

[7] G.Aielli, these proceedings

[8] ATLAS Collaboration, Technical Design Report for the Phase-II Upgrade of the ATLAS Muon Spectrometer,

https://cds.cern.ch/record/2285580 